\title{
COVID-19 Vaccination in Patients with Chronic Lymphocytic Leukaemia: What Can We Expect?
}

\author{
Mohammad Ammad Ud Din and Saad Jamshed² \\ 1. Internal Medicine, Rochester General Hospital, Rochester, New York, USA; 2. Hematology/Oncology, Rochester General Hospital, \\ Rochester, New York, USA.
}

DOI: https://doi.org/10.17925/OHR.2021.17.1.7

$\mathrm{P}$ atients with chronic lymphocytic leukaemia are at an increased risk of developing severe coronavirus disease (COVID-19). Although rollout of the novel vaccines against the disease may offer hope to ending the pandemic, their efficacy in patients with chronic lymphocytic leukaemia may be significantly lower compared to the general population.

\section{Keywords}

COVID-19 vaccine, chronic lymphocytic leukemia, mRNA vaccine, coronavirus, pandemic

Disclosures: Mohammad Ammad Ud Din and Saad Jamshed have no financial or non-financial relationships or activities to declare in relation to this article.

Review Process: Double-blind peer review

Compliance with ethics: This article is an opinion piece and does not report on new clinical data, or any studies with human or animal subjects performed by any of the authors.

Authorship: The named authors meet the International Committee of Medical Journal Editors (ICMJE) criteria for authorship of this manuscript, take responsibility for the integrity of the work as a whole, and have given final approval for the version to be published. Access: This article is freely accessible at touchONCOLOGY.com (c) Touch Medical Media 2021.

Submitted: 7 January 2021

Accepted: 12 February 2021

Published online: 19 March 2021

Citation: touchREVIEWS in Oncology \& Haematology. 2021;17(1):7-8

Corresponding author: Mohammad Ammad Ud Din Internal Medicine, Rochester General Hospital, 1425 Portland Avenue, Rochester, New York, NY 14621, USA. E:ammadahr@gmail.com

Support: No funding was received for the publication of this article.
Advanced age and other comorbid conditions put patients with chronic lymphocytic leukaemia (CLL) at an increased risk of morbidity and mortality from SARS-COV-2 infection, with early studies showing case fatality rates of over $30 \%$ in patients with CLL with symptomatic COVID-19.1,2 As the vaccination against COVID-19 is becoming available for use in the general population, its efficacy in conditions with dysfunctional humoral immunity, such as CLL, is unclear.

Multiple studies have shown a subpar response to vaccination against common respiratory pathogens like Streptococcus pneumoniae, Haemophilus influenzae and the influenza virus in patients with CLL. ${ }^{3-5}$ However, despite the lower rate of seroconversion, vaccines remain one of the cornerstones for infection prevention, and international societies favour their use as they are generally safe. ${ }^{6,7}$ The perturbation of humoral immunity is a cardinal feature of CLL that worsens with disease progression. The pathogenesis is complex and involves impaired signalling between $\mathrm{B}$ and T cells, and B-CLL cell-mediated plasma cell apoptosis through CD95-CD95L interaction leading to hypogammaglobulinaemia. ${ }^{8}$ Several treatment options, such as anti-CD20 antibodies and Bruton tyrosine kinase (BTK) inhibitors, have also been shown to cause hypogammaglobulinaemia through various intracellular mechanisms.8-10

Apart from the subnormal humoral immunity, deranged cellular immunity resulting from impaired actin polymerisation in $\mathrm{CD} 4+$ and $\mathrm{CD} 8+\mathrm{T}$ cells also plays an important role in the poor response to vaccinations as these molecular changes lead to the subsequent inability to form effective synapses with antigen-presenting cells. ${ }^{11}$ Although some studies show paradoxical partial immune restitution with the use of BTK inhibitors with patients with COVID-19 benefiting from the use of ibrutinib, generally immune dysfunction worsens with the progression of the disease and the use of chemoimmunotherapy or novel targeted agents. The literature supports early vaccination of these patients for better results. $4,5,12$

The new generation COVID-19 vaccines utilize a novel technique of nano lipid particles instead of an attenuated vector for the delivery of the messenger RNA (mRNA) coding for the surface spike protein (S protein) of SARS-COV-2. ${ }^{13}$ However, the eventual production of antibodies against the $S$ protein is dependent on healthy T-helper and B-cell interaction and an overall functional humoral immune system. The phase III trial that formed the basis for the approval of the Pfizer/BioNTech and Moderna vaccines excluded immunocompromised individuals with known or suspected immunodeficiency. ${ }^{13,14}$ Although data are currently scarce, it is likely the mRNA vaccines may not cause additional side effects in patients with CLL compared with the general population as they do not have a "live" component. Even vaccines using an attenuated adenovirus, such as that of AstraZeneca/Oxford, will hopefully be safe as the viral reproductive genetic region is spliced out to include the S protein gene..$^{15}$ Previous studies have also consistently shown that the influenza vaccine is safe for use in haematological malignancies, including patients with CLL. ${ }^{16}$

In terms of efficacy, based on the extrapolation of data available from other vaccines, treatmentnaive patients who are early on in their disease course will likely show a better serological response. However, the response rate might still be lower than the general population as they still 
have some degree of disease-related immune dysfunction. The duration of protection offered by these vaccines is still unknown for the general population, but their durability of action might be shorter in comparison to patients with CLL as the rate of antigen-specific immunoglobulin titres have been shown to fall quicker over time in individuals with haematological malignancies compared with healthy controls. ${ }^{2,17}$

With time, as the global vaccination drive against COVID-19 gains pace, more information will be available regarding the efficacy of these vaccines in patients who are immunocompromised or those with cancer. At the moment, the Centers for Disease Control and Prevention (CDC) recommendations do not specifically comment on patients with $\mathrm{CLL}$, but do state that immunocompromised individuals can receive the vaccination if they do not have other contraindications to a specific component of the vaccines. ${ }^{18}$ The American Society of Clinical Oncology and the American Society of Hematology also recommend shared decision making with patients on this topic, keeping in mind the incidence of infection in the community and assessing the individual risk of severe infection on a case-by-case basis. ${ }^{19,20}$

Although the CDC recommends delaying vaccination for 3 months following confirmed COVID-19, as reinfection is uncommon in the 90 days following the initial infection, early studies have shown a precipitous decline in protective antibodies in the few weeks following SARS-CoV-2 infection in patients with CLL., ${ }^{2,18}$ Therefore, it is important to risk stratify patients rather than generalize the postponement of vaccination in every case..$^{20}$ Ideally, patients should be considered for vaccination 2-4 weeks before the initiation of immunosuppressive therapy, or 6 months after completion of treatment to improve the chances of an adequate serological response, but a delay of 6 months may be impractical in light of the pandemic. ${ }^{20}$ Furthermore, even though the number of haematopoietic cell transplantations for the treatment of CLL has gone down because of the mainstream use of novel agents, transplant patients also can be vaccinated 3-6 months post-procedure based on data regarding response to other vaccines. ${ }^{20}$

These recommendations may change as we learn more in the coming year, but for now, to help with making an informed decision, patients with CLL should be counselled about having no increased risk of side effects to the vaccines. The main shortcoming may be the possibility of a weaker immunologic response. If a decision is made to vaccinate, it is important to adhere to the twice-dosing schedule, and the same kind of vaccine should be used for both doses. ${ }^{20}$ In high-risk patients, specific-IgG titres can be checked 4 weeks after vaccination to assess response. Currently, only state-level laboratories can perform such testing as most assays for anti-S antibodies are reserved for clinical trials only, and it will take time for them to become widely available for commercial use. ${ }^{21}$

As patients with CLL are prone to infections, it also remains of critical significance to educate them regarding the continued need to follow preventive measures, such as social distancing, hand washing and face coverings, as advised by their local health authorities, regardless of their vaccination status.
1. Mato AR, Roeker LE, Lamanna M, et al. Outcomes of COVID-19 in patients with CLL: a multicenter international experience. Blood. 2020;13:1134-43.

2. Roeker LE, Knorr DA, Pessin MS, et al. Anti-SARS-CoV-2 antibody response in patients with chronic lymphocytic leukemia. Leukemia. 2020;34:3047-9.

3. van der Velden AM, Mulder AH, Hartkamp A, et al. Influenza virus vaccination and booster in B-cell chronic lymphocytic leukaemia patients. Eur J Intern Med. 2001:12:420-4.

4. Hartkamp A, Mulder AH, Rijkers GT, et al. Antibody responses to pneumococcal and haemophilus vaccinations in patients with B-cell chronic lymphocytic leukaemia. Vaccine. 2001;19:1671-7.

5. Lindström V, Aittoniemi J, Salmenniemi U, et al. Antibody response to the 23-valent pneumococcal polysaccharide vaccine after conjugate vaccine in patients with chronic lymphocytic leukemia. Hum Vaccin Immunother. 2019;15:2910-13.

6. Bridges $C B$, Woods $L$, Coyne-Beasley T. Advisory Committee on Immunization Practices (ACIP). Recommended immunization schedule for adults aged 19 years and older - United States. 2013. Available at: www.cdc.gov/mmwr/preview/mmwrhtml/ 2013. Avallable at: Www.cdc.gov/mmwr/p
su6201a3.htm (accessed 3 January 2021).

7. Eichhorst B, Robak T, Montserrat T, et al. Chronic lymphocytic leukaemia: ESMO Clinical Practice Guidelines for diagnosis, leukaemia: ESMO Clinical Practice Guidelines for diagnos

8. Grywalska E, Zaborek M, tyczba J, et al. Chronic lymphocytic leukemia-induced humoral immunosuppression: a systematic review. Cells. 2020;9:2398.

9. Andrick B, Alwhaibi A, DeRemer DL, et al. Lack of adequate pneumococcal vaccination response in chronic lymphocytic leukaemia patients receiving ibrutinib. Br J Haematol. 2018;182:712-4.

10. Douglas AP, Trubiano JA, Barr I, et al. Ibrutinib may impair serological responses to influenza vaccination. Haematologica. 2017:102:e397-9.

11. Ramsay AG, Johnson AJ, Lee AM, et al. Chronic lymphocytic leukemia T cells show impaired immunological synapse formation that can be reversed with an immunomodulating drug. I Clin Invest. 2008; 118:2427-37.

12. Scarfò L, Chatzikonstantinou T, Rigolin GM, et al. CovID-19 severity and mortality in patients with chronic lymphocytic leukemia: a joint study by ERIC, the European Research Initiative on CLL, and CLL Campus. Leukemia. 2020; 34:2354-63.

13. Polack FP, Thomas SJ, Kitchin N, et al. Safety and efficacy of the BNT162b2 mRNA Covid-19 vaccine. N Eng/ J Med. 2020;383:2603-15

14. Baden $L R$, El Sahly HM, Essink B, et al. Efficacy and safety of the mRNA-1273 SARS-CoV-2 vaccine. N Eng/ J Med. 2021:384:403-16.

15. Voysey M, Costa Clemens SA, Madhi SA, et al. Safety and efficacy of the ChAdOx1 nCoV-19 vaccine (AZD1222) against SARS-COV-2: an interim analysis of four randomised controlled trials in Brazil, South Africa, and the UK. Lancet. 2021;397:99-111.
16. La Torre G, Mannocci A, Colamesta V, et al. Influenza and pneumococcal vaccination in hematological malignancies a systematic review of efficacy, effectiveness, and safety. Mediterr I Hematol Infect Dis. 2016;8:e2016044.

17. Mustafa SS, Shah D, Bress J, Jamshed S. Response to PCV13 vaccination in patients with multiple myeloma versus healthy controls. Hum Vaccin Immunother. 2019;15:452-4.

18. Centers for Disease Control and Prevention. Interim clinical considerations for use of Pfizer-BioNTech COVID-19 vaccine. 2020. Available at: www.cdc.gov/vaccines/covid-19/info-byproduct/clinical-considerations.html (accessed 5 January 2021).

19. American Society of Clinical Oncology. COVID-19 vaccine \& patients with cancer. 2020. Available at: www.asco.org/ asco-coronavirus-resources/covid-19-vaccines-patients-cancer (accessed 18 March 2021).

20. American Society of Hematology, American Society for Transplantation and Cellular Therapy. COVID-19 and Vaccines for the Immunocompromised: Frequently Asked Questions. 23 December 2020 Available at: mum hematology/org/covid-19/ December 2020. Available at. www.hematology.org/covid-19/

21. Labcorp. LabCorp launches quantitative antibody test to assess effectiveness of COVID-19 vaccines in clinical trials. 2020. Available at: www.labcorp.com/coronavirus-disease-covid-19/ news/labcorp-launches-quantitative-antibody-test-assessnews/labcorp-launches-quantitative-antibody-test-assess-
effectiveness-covid-19-vaccines-clinical-trials (accessed 18 March 2021). 